\title{
Functional analysis of a Campylobacter jejuni alkaline phosphatase secreted via the Tat export machinery
}

\author{
Andries van Mourik, Nancy M. C. Bleumink-Pluym, Linda van Dijk, \\ Jos P. M. van Putten and Marc M. S. M. Wösten
}

Correspondence

Marc M. S. M. Wösten

M.Wosten@uu.nl

Received 29 July 2007

Revised 2 November 2007

Accepted 6 November 2007 Department of Infectious Diseases and Immunology, Utrecht University, Yalelaan 1,
3584 CL Utrecht, The Netherlands

\section{INTRODUCTION}

The human pathogen Campylobacter jejuni is one of the most important causes of bacterial gastroenteritis worldwide (Altekruse et al., 1999). The natural habitat of C. jejuni is the intestine of warm-blooded animals and a wide variety of watery environmental sources (Diergaardt et al., 2004; Rosef et al., 2001). This diversity in ecological niche demands that the bacterium is able to adapt rapidly to changing environments, such as alterations in temperature or the availability of oxygen and nutrients. In recent years a number of signal transduction systems have been identified in C. jejuni that may contribute to bacterial adaptation (MacKichan et al., 2004). One two-component signal transduction system important for bacterial survival is the PhosS-PhosR system, which senses changes in phosphate concentration and responds by altering the expression of a number of genes (the pho regulon) involved in phosphate acquisition and diverse metabolic processes (Wösten et al., 2006).

For most bacterial species, inorganic phosphate $\left(\mathrm{P}_{\mathrm{i}}\right)$ is the preferred source of phosphate. Most bacteria assimilate $\mathrm{P}_{\mathrm{i}}$

Abbreviation: PNPP, p-nitrophenyl phosphate. via specific uptake systems after hydrolysis of phosphatecontaining substrates in the periplasm. An important enzyme involved in the hydrolysis of a variety of exogenous phosphate sources is alkaline phosphatase (PhoA). This metalloenzyme catalyses the non-specific hydrolysis of phosphomonoesters to an alcohol and $\mathrm{P}_{\mathrm{i}}$ and thus makes $\mathrm{P}_{\mathrm{i}}$ available for utilization by the bacterium. The production of PhoA is regulated by the phosphate concentration in the environment and upregulated during phosphate limitation. The enzyme is transported into the periplasm via the general secretory $(\mathrm{Sec})$ system and becomes active after dimerization and binding of two $\mathrm{Zn}^{2+}$ and one $\mathrm{Mg}^{2+}$ cations (Torriani, 1990; Wang et al., 2005).

Intriguingly, search of the whole genome of $C$. jejuni for phoA homologues suggested that the pathogen lacks the typical PhoA. Characterization of the pho regulon of $C$. jejuni, however, indicated that the bacterium possesses a phosphatase that is involved in phosphate assimilation (Wösten et al., 2006). The corresponding gene (Cj0145) was upregulated during phosphate limitation and under the control of the PhosS-PhosR two-component system (Wösten et al., 2006). To further decipher the nature of this seemingly atypical enzyme, which was provisionally 
designated $\mathrm{PhoA}^{\mathrm{Cj}}$, we further investigated the regulation, transport, activation and function of the enzyme. Here we provide evidence that the $C$. jejuni phosphatase deviates from the classical Escherichia coli PhoA in that it is transported over the cytoplasmic membrane via a previously unidentified twin-arginine translocation (Tat) secretion system, requires $\mathrm{Ca}^{2+}$ for its activity, and exclusively utilizes phosphomonoesters as a substrate.

\section{METHODS}

Bacterial strains and culture conditions. The strains and plasmids used in this study are listed in Table 1. C. jejuni strains were routinely cultured under microaerophilic conditions $\left(5 \% \mathrm{O}_{2}, 10 \% \mathrm{CO}_{2}\right.$ and $85 \% \mathrm{~N}_{2}$ ) at $37{ }^{\circ} \mathrm{C}$ on plates containing blood agar base II (Oxoid) and $5 \%$ horse blood lysed with $0.5 \%$ saponin (Sigma) or in Heart Infusion broth (HI) (Oxoid). E. coli strains were grown on LuriaBertani (LB) agar plates or in LB medium at $37^{\circ} \mathrm{C}$. When appropriate, media were supplemented with ampicillin $(100 \mu \mathrm{g}$ $\left.\mathrm{ml}^{-1}\right)$, kanamycin $\left(50 \mu \mathrm{g} \mathrm{ml}^{-1}\right)$ or chloramphenicol $\left(20 \mu \mathrm{g} \mathrm{ml}^{-1}\right)$.
Construction of a C. jejuni tatC mutant. To inactivate tatC (Cj0578), the gene and its flanking genes were amplified with the primers TAT-1 and TAT-2 (Table 2) using C. jejuni 81116 chromosomal DNA as template. The resulting $2181 \mathrm{bp}$ PCR product was cloned into the pGEM-T easy vector (Promega) to form plasmid pGEM578. Inverse PCR was performed to introduce a deletion of $424 \mathrm{bp}$ in the tatC gene and to create an unique BamHI restriction site by using the primers TAT-3BamHI and TAT-4BamHI (Table 2). After self-ligation, the plasmid was digested with BamHI and ligated to the $\mathrm{Km}^{\mathrm{r}}$ cassette gene of pILL550 (Labigne-Roussel et al., 1987). Natural transformation (Wassenaar et al., 1993) was used to introduce the resulting knockout plasmid pGEM578:: Km into $C$. jejuni to obtain the 81116 tatC:: Km mutant.

Construction of complementation plasmids. To complement the $p h o A^{\mathrm{Cj}}:: \mathrm{Cm}$ (Wösten et al., 2006) and tatC:: Km mutants, two $E$. coli-C. jejuni shuttle conjugative expression plasmids, pMA1 and pMA2, were constructed. To this end, the C. jejuni metK (Cj1096) promoter was amplified with the primers metKFSphI and metKRNsiI (Table 2). After digestion with SphI and NsiI, the resulting $115 \mathrm{bp}$ PCR product was ligated into SphI- and NsiI-digested pWM1007 (Miller et al., 2000), yielding plasmid pWM1007-pr.metK. The metK

Table 1. Bacterial strains and plasmids used in this study

\begin{tabular}{|c|c|c|}
\hline Strain or plasmid & Genotype or relevant characteristics & Source or reference \\
\hline \multicolumn{3}{|l|}{ C. jejuni strains } \\
\hline 81116 & Wild-type & Palmer et al. (1983) \\
\hline NCTC 11168 & Wild-type & Parkhill et al. (2000) \\
\hline 480 & Wild-type & Wassenaar et al. (1993) \\
\hline 81116 tatC: $: \mathrm{Km}$ & 81116 derivative tat $C:: \mathrm{Km}$ & This study \\
\hline 81116tatC: : Km + pMA2-tatC & 81116 derivative tat $C:: \mathrm{Km}+\mathrm{pMA} 2$-tatC & This study \\
\hline $81116 p h o A^{\mathrm{Cj}}:: \mathrm{Cm}$ & 81116 derivative $p h o A^{\mathrm{Cj}}:: \mathrm{Cm}$ & Wösten et al. (2006) \\
\hline $81116 p h o A^{\mathrm{Cj}}:: \mathrm{Cm}+\mathrm{pMA} 1$ & 81116 derivative $p h o A^{\mathrm{Cj}}:: \mathrm{Cm}+\mathrm{pMA} 1$ & This study \\
\hline $81116 p h o A^{\mathrm{Cj}}:: \mathrm{Cm}+$ pMAl-phoA $^{\mathrm{Cj}}$ & 81116 derivative $p h o A^{\mathrm{Cj}}:: \mathrm{Cm}+\mathrm{pMA}^{-}-\mathrm{phoA}^{\mathrm{Cj}}$ & This study \\
\hline $81116 p h o A^{\mathrm{Cj}}:: \mathrm{Cm}+$ pMA1-GGphoA $^{\mathrm{Cj}}$ & 81116 derivative $p h o A^{\mathrm{Cj}}:: \mathrm{Cm}+$ pMAl-GGphoA $^{\mathrm{Cj}}$ & This study \\
\hline $81116 p h o A^{\mathrm{Cj}}:: \mathrm{Cm}+$ pMA1-phoA $^{\mathrm{Ec}}$ & 81116 derivative $p h o A^{\mathrm{Cj}}:: \mathrm{Cm}+\mathrm{pMAl}^{-\mathrm{phoA}^{\mathrm{Ec}}}$ & This study \\
\hline \multicolumn{3}{|l|}{ E. coli strain } \\
\hline PC2955 & $\begin{array}{l}\text { relA1 } \phi 80 \mathrm{~d} l a c Z \Delta \mathrm{M} 15 \text { phoA8 hsdR17 recA1 endAl gyrA96 thi-1 } \\
\text { relA1 luxS glnV44 }\end{array}$ & NCCB \\
\hline \multicolumn{3}{|l|}{ Plasmids } \\
\hline pGEM-T easy & PCR cloning vector, $\mathrm{Amp}^{\mathrm{r}}$ & Promega \\
\hline pGEM578 & pGEM-T easy containing tatC & This study \\
\hline pILL550 & Shuttle cloning vector & Labigne-Roussel et al. (1987) \\
\hline pBluescript KS M13+ & Cloning vector, $\mathrm{Amp}^{\mathrm{r}}$ & Stratagene \\
\hline pCB267 & lacZ and phoA promoter probe vector & Schneider \& Beck (1986) \\
\hline pGEM145 & pGEM-T easy containing $p h o A^{\mathrm{Cj}}$ & This study \\
\hline pGEM145GG & pGEM145 containing a RR substituted $p h o A^{\mathrm{Cj}}$ gene & This study \\
\hline pGEMphoA & pGEM-T easy containing E. coli phoA & This study \\
\hline pGEM578-2 & pGEM-T easy containing tat $C$ & This study \\
\hline pMA1 & C. jejuni/E. coli shuttle conjugative expression vector, $\mathrm{Km}^{\mathrm{r}}$ & This study \\
\hline pMA2 & C. jejuni/E. coli shuttle conjugative expression vector, $\mathrm{Cm}^{\mathrm{r}}$ & This study \\
\hline pWM1007 & C. jejuni/E. coli shuttle vector & Miller et al. (2000) \\
\hline pWM1007-pr.metK & pWM1007 containing the metK promoter & This study \\
\hline pAV35 & pBluescript II SK containing E. coli $\mathrm{Cm}^{\mathrm{r}}$ cassette & van Vliet et al. (1998) \\
\hline pMA1-phoA ${ }^{\mathrm{Cj}}$ & pMA1 containing $p h o A^{\mathrm{Cj}}$ & This study \\
\hline pMA1-GGphoA ${ }^{\mathrm{Cj}}$ & pMA1 containing a RR substituted $p h o A^{\mathrm{Cj}}$ & This study \\
\hline pMA1-phoA ${ }^{\mathrm{Ec}}$ & pMA1 containing E. coli phoA & This study \\
\hline pMA2-tatC & pMA2 containing $C$. jejuni tat $C$ & This study \\
\hline
\end{tabular}


Table 2. Primers used in this study

\begin{tabular}{|ll|}
\hline Primer & \multicolumn{1}{c|}{ DNA sequence $\mathbf{( 5}^{\prime}-\mathbf{3}^{\prime} \mathbf{)}$} \\
\hline TAT-1 & AAATTTAGAAGGCGGGCGTGTT \\
TAT-2 & GCAAAAATTCTAAAGGCTGTAAA \\
TAT-3BamHI & AAAGGATCCTTCATTGCGGTAAATAAGGC \\
TAT-4BamHI & AAAGGATCCTCAATTTTAATGGCAGGACC \\
phoAF1 & GAGCTCAGGAGAAAAAACAAATGGAAAGAAGA \\
phoAF2 & GAGCTCAGGAGAAAAAACAAATGGAAGGAGGATTGTTT \\
phoAR & CCGCGGTATTTAGCTTCCTATCACTCCAC \\
phoAECF & GAGCTCAGGAGAAAATAAAATGAAACAAA \\
phoAECR & CCGCGGGGTATCAGGCGGCTTTCTTGAGG \\
TATcplforw.xbaI & TCTAGAGTGTAATCATAGCTTGAAAG \\
TATcplrev1.sacI & GAGCTCCAAGATAAAAACGGAAAAATAAATG \\
metKFSphI & GCATGCAGTTGATTTTAACTAACTTTTGCT \\
metKRNsiI & ATGCATAAAAAGTCCTTTCATTTAAAATG \\
pILL550-pWM1007F & CCAGATCTGATGATAAGCTGTCAAACATGAG \\
pILL550-pWM1007R & CCGAATTCAGCGACCGGCGCTCAGCTGG \\
T7 & TAATACGACTCACTATAGGG \\
22CATDO & CTGGGATTTTATTTATTCAGCAAG \\
\hline
\end{tabular}

promoter, polylinker and the gene coding for green fluorescent protein (GFP) were amplified from pWM1007-pr.metK with the primers pILL550-pWM1007F and pILL550-pWM1007R (Table 2) and the $1.9 \mathrm{~kb}$ product was digested with BglII and EcoRI. The shuttle conjugative expression plasmid pMA1 was obtained by ligation of this $1.9 \mathrm{~kb}$ fragment into the BglII/EcoRI-digested plasmid pILL550. Plasmid pMA2 contains a chloramphenicol cassette $\left(\mathrm{Cm}^{\mathrm{r}}\right)$ instead of the kanamycin resistance gene. For construction of this plasmid, the $\mathrm{Cm}^{\mathrm{r}}$ of pAV35 (van Vliet et al., 1998) was amplified with the primers T7 and 22CATDO (Table 2). The PCR product of $880 \mathrm{bp}$ containing one EcoRI site was digested with EcoRI and ligated into an EcoRI- and SwaI-digested pMA1, resulting in plasmid pMA2.

To complement the tatC::Km mutant, the tatC gene was amplified with the primers TATcplforw.xbaI and TATcplrevl.sacI (Table 2). The restriction enzymes SacI and $\mathrm{XbaI}$ were used to clone the $800 \mathrm{bp}$ tatC PCR fragment into pMA2, generating the complementation plasmid pMA2-tatC.

Plasmid pMA1 was used to introduce the $p h o A^{\mathrm{Cj}}$, the GGphoA $A^{\mathrm{Cj}}$ construct (a gene coding for a $\mathrm{PhoA}{ }^{\mathrm{Cj}}$ protein where the twin arginine residues are substituted by glycine residues) and an E. coli phoA gene into the C. jejuni phoA::Cm mutant. By using the primer combinations phoAF1/phoAR and phoAF2/phoAR (Table 2) and $C$. jejuni 81116 chromosomal DNA as template, $p h o A^{\mathrm{Cj}}$ and GGphoA ${ }^{\mathrm{Cj}}$ PCR products were generated. These PCR products of $1800 \mathrm{bp}$ in size were digested with SacI and SacII and ligated into pMA1, resulting in the plasmids pMA1-phoA ${ }^{\mathrm{Cj}}$ and pMA1-GGphoA ${ }^{\mathrm{Cj}}$, respectively. The phoA gene of E. coli was PCR amplified from pCB267 (Schneider \& Beck, 1986) with the primers phoAECF/phoAECR (Table 2). The $1800 \mathrm{bp}$ PCR product was digested with SacI and SacII and introduced into pMA1 to form plasmid pMA1-phoA ${ }^{\mathrm{Ec}}$. All PCR products in this study were obtained with the proofreading enzyme $P f u$ (Promega) according to the instructions of the manufacturer. Nucleotide sequences of the cloned PCR products were verified by sequencing both strands. Complementation plasmids were introduced into C. jejuni mutants via conjugation (Labigne-Roussel et al., 1987).

Alkaline phosphatase assay. Alkaline phosphatase activity was determined as previously described (Wösten et al., 2006), except that EDTA was omitted from the lysis buffer. In short, alkaline phosphatase activity of a bacterial culture grown in defined medium (Leach et al., 1997) containing $1.6 \mathrm{mM} \mathrm{P}_{\mathrm{i}}$ (high) or $0.08 \mathrm{mM} \mathrm{P}_{\mathrm{i}}$ (low) was assayed by monitoring the release of $p$-nitrophenol from $p$-nitrophenyl phosphate (PNPP) (Sigma). The units of alkaline phosphatase were calculated using the formula $10^{3} \times\left[A_{420}-\left(1.75 \times A_{550}\right)\right] / t \times \mathrm{OD}_{600} \times V$.

Nitrite assay. Nitrate reductase activity was determined as previously described (Sellars et al., 2002). Briefly, C. jejuni strains were grown overnight in $\mathrm{HI}$ medium containing $50 \mathrm{mM}$ potassium nitrate. To the culture supernatants $1 \%(\mathrm{w} / \mathrm{v})$ sulphanilamide dissolved in $1 \mathrm{M} \mathrm{HCl}$ and $0.02 \%(\mathrm{w} / \mathrm{v})$ naphthylethylenediamine were added and after $15 \mathrm{~min}$ absorbance was measured at $540 \mathrm{~nm}$. Nitrite concentrations, adjusted to the cell density, were determined by reference to a standard curve.

Growth experiments. Overnight cultures grown in HI medium or defined medium were diluted to a starting $\mathrm{OD}_{600}$ of 0.05 and grown at $37{ }^{\circ} \mathrm{C}$ under microaerophilic $\left(5 \% \mathrm{O}_{2}\right)$ or oxygen-limited $(0.3 \%$ $\mathrm{O}_{2}$ ) conditions using the anoxomat system (MART Microbiology $\mathrm{BV})$. Bacterial growth and cell density were monitored by measuring $\mathrm{OD}_{600}$ and counting c.f.u. at intervals.

Localization of the alkaline phosphatase activity. A $10 \mathrm{ml}$ C. jejuni culture grown with $0.08 \mathrm{mM} \mathrm{P}_{\mathrm{i}}$ was subjected to cellular fractionation as described by Myers \& Kelly (2005).

\section{RESULTS}

\section{Regulation of PhoA ${ }^{{ }^{C j}}$ phosphatase activity}

Inactivation of gene $\mathrm{Cj} 0145$ encoding $\mathrm{PhoA}^{\mathrm{Cj}}$ in $C$. jejuni strain 81116 resulted in a complete loss of bacterial phosphatase activity (Fig. 1) (Wösten et al., 2006). To ensure that this phenotype was caused by disruption of PhoA ${ }^{\mathrm{Cj}}$, we complemented the $p h o A^{\mathrm{Cj}}: \mathrm{Cm}$ mutant in trans with the shuttle plasmid pMA1-phoA ${ }^{\mathrm{Cj}}$. This plasmid constitutively expresses the C. jejuni phoA gene via the $m e t K$ promoter. Plasmid $\mathrm{pMA}^{-}-\mathrm{phoA}^{\mathrm{Cj}}$ restored the 


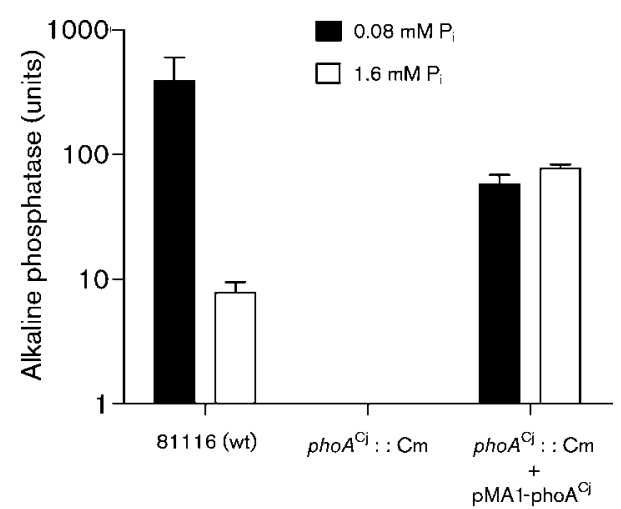

Fig. 1. Phosphatase $\left(P h o A^{C j}\right)$ activity of $C$. jejuni strains during phosphate starvation. Alkaline phosphatase activity in bacterial whole-cell lysates was measured for strain 81116 wild-type, the pho $A^{\mathrm{Cj}}:: \mathrm{Cm}$ mutant strain, and the $p h \circ A^{\mathrm{Cj}}:: \mathrm{Cm}$ mutant strain containing the complementation plasmid $\mathrm{pMA} 1-\mathrm{PhoA}^{\mathrm{Cj}}$. Bacteria were grown in defined medium containing 1.6 or $0.08 \mathrm{mM} \mathrm{P}_{\mathrm{i}}$. Standard errors based on four independent experiments are shown.

phosphatase activity when bacteria were grown in lowphosphate medium (Fig. 1, filled bars), indicating that $\mathrm{PhoA}^{\mathrm{Cj}}$ is responsible for the phosphatase activity.

To investigate whether additional components induced under conditions of low $\mathrm{P}_{\mathrm{i}}$ were required for $\mathrm{PhoA}^{\mathrm{Cj}}$ activity, we compared the enzyme activity for the wildtype, mutant and complemented strains after growth in low- and high-phosphate conditions. Whereas for the wildtype enzyme activity clearly varied with the availability of phosphate in the medium, this regulatory effect was lost in the complemented strain in which $\mathrm{PhoA}^{\mathrm{Cj}}$ was constitutively expressed (Fig. 1). This indicates that, at least when the gene is expressed from a multicopy plasmid, no additional components present under low-phosphate conditions are necessary for the $\mathrm{PhoA}^{\mathrm{Cj}}$ activity.

\section{pH optimum of C. jejuni phosphatase activity}

To assess whether $\mathrm{PhoA}^{\mathrm{Cj}}$ is an alkaline phosphatase, we measured the phosphatase activity at different $\mathrm{pH}$ values. Wild-type 81116 and the $p h o A^{\mathrm{Cj}}:: \mathrm{Cm}$ mutant were grown in defined medium under low-phosphate conditions and lysed in Tris/HCl buffer containing SDS and lysozyme with different $\mathrm{pH}$ values. Maximum phosphatase activity was obtained at pH 10 (Fig. 2a). No phosphatase activity was observed for the $p h o A^{\mathrm{Cj}}:: \mathrm{Cm}$ mutant over the entire $\mathrm{pH}$ range (data not shown), indicating that $\mathrm{PhoA}^{\mathrm{Cj}}$ is the sole phosphomonoesterase of $C$. jejuni.

\section{PhoA ${ }^{\mathrm{Cj}}$ activity requires $\mathrm{Ca}^{2+}$}

Assessment of the conservation of alkaline phosphatase activity among $C$. jejuni strains revealed considerable variation in enzyme activity (Fig. 2b), although they all contained an intact $p h o A^{\mathrm{Cj}}$ gene (data not shown). One explanation for this diversity may be the variable presence of enzyme cofactors. As alkaline phosphatases of other species require divalent cations for their activity, we tested C. jejuni phosphatase activity in bacteria lysed in the absence of the metal chelator EDTA. All C. jejuni strains tested showed a strong increase in alkaline phosphatase activity in the absence of EDTA (Fig. 2b), indicating that $\mathrm{PhoA}^{\mathrm{Cj}}$ needs a cation for its activity.

The nature of the cation that activates the $\mathrm{PhoA}^{\mathrm{Cj}}$ was sought by selective addition of cations to bacteria grown overnight in the presence of $1 \mathrm{mM}$ EDTA (Fig. 2c). Enzyme activity was measured $30 \mathrm{~min}$ after the addition of the cation(s) to the culture. Alkaline phosphatase activity in the EDTA-treated strains was almost fully restored by the addition of $10 \mathrm{mM} \mathrm{Ca}^{2+}$, while other ions including $\mathrm{Mn}^{2+}, \mathrm{K}^{+}, \mathrm{Mg}^{2+}, \mathrm{Zn}^{2+}$ (Fig. 2c) or $\mathrm{Na}^{+}, \mathrm{Cu}^{2+}, \mathrm{Co}^{2+}$, $\mathrm{Ni}^{2+}, \mathrm{Mo}^{6+}, \mathrm{VO}_{4}^{3-}, \mathrm{Fe}^{2+}$ or $\mathrm{Fe}^{3+}$ or combinations thereof (data not shown), did not restore enzyme activity.

\section{Substrate specificity of $C_{\text {. jejuni PhoA }}{ }^{C_{j}}$}

The main function of bacterial alkaline phosphatases is to release $P_{i}$ from various exogenous organophosphate compounds for use in bacterial growth. To assess which type of compounds may serve as a substrate for $\mathrm{PhoA}^{\mathrm{Cj}}$, we tested various potential phosphate donors for their ability to support growth of $C$. jejuni 81116 and the $p h o A^{\mathrm{Cj}}:: \mathrm{Cm}$ mutant strain. Measurement of the optical density and c.f.u. counting of overnight cultures showed that the wildtype strain but not the mutant grew with $1.6 \mathrm{mM}$ of the organophosphate monoesters, glucose 6-phosphate (G6P) and glycerol 3-phosphate (G3P) as sole phosphate source, while no growth was observed with the phosphodiesters cAMP or DNA (Fig. 2d).

\section{PhoA ${ }^{C j}$ requires a twin-arginine sequence for enzyme activity}

A large number of bacterial alkaline phosphatases are secreted via the Sec-dependent pathway. Analysis of the $\mathrm{PhoA}^{\mathrm{Cj}}$ protein revealed that it contains a conserved twinarginine domain near the $\mathrm{N}$ terminus, characteristic of proteins that are exported by the Tat secretion machinery (Stanley et al., 2000). To address whether PhoA ${ }^{\mathrm{Cj}}$ might be transported by the C. jejuni Tat system, we replaced the coding sequence for two arginine residues with two glycine residues in the $p h o A^{\mathrm{Cj}}$ located on plasmid pMA1-phoA ${ }^{\mathrm{Cj}}$, containing the constitutively expressed metK promoter. The resulting plasmid pMAl-GGphoA ${ }^{\mathrm{Cj}}$ was introduced into $p h o A^{\mathrm{Cj}}:: \mathrm{Cm}$ mutant strain and alkaline phosphatase activity was measured under high- and low-phosphate concentrations. This strain lacked alkaline phosphatase activity under conditions of phosphate limitation, while minimal activity (less than $5 \%$ of wild-type) was observed under high-phosphate conditions (Fig. 3a). For the control 
(a)

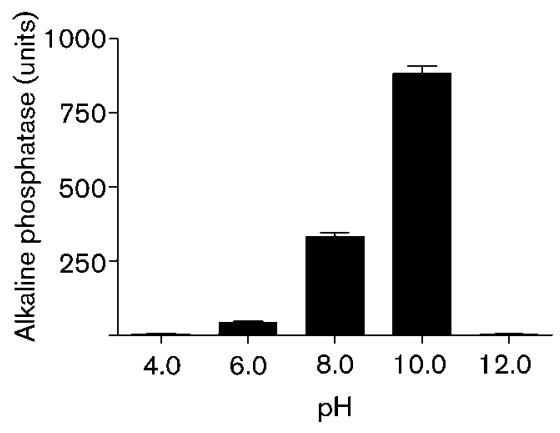

(c)

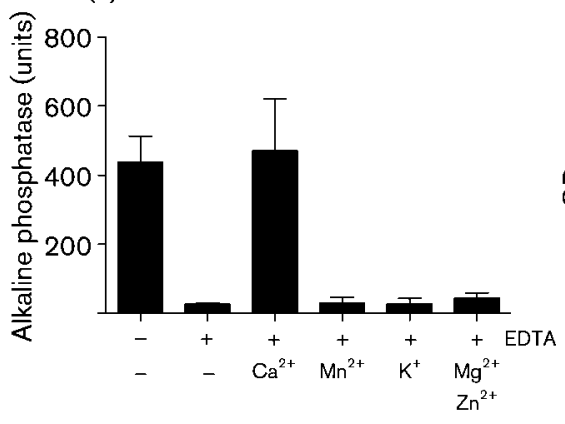

(b)

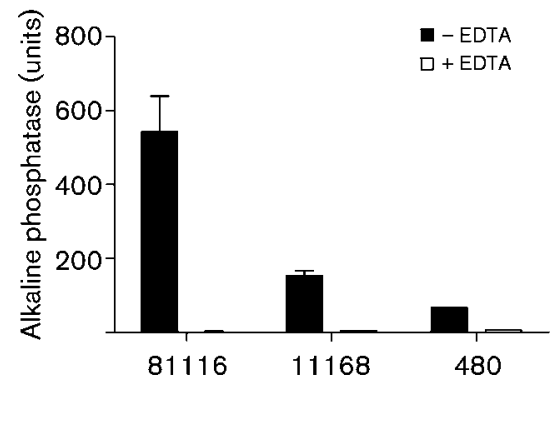

(d)

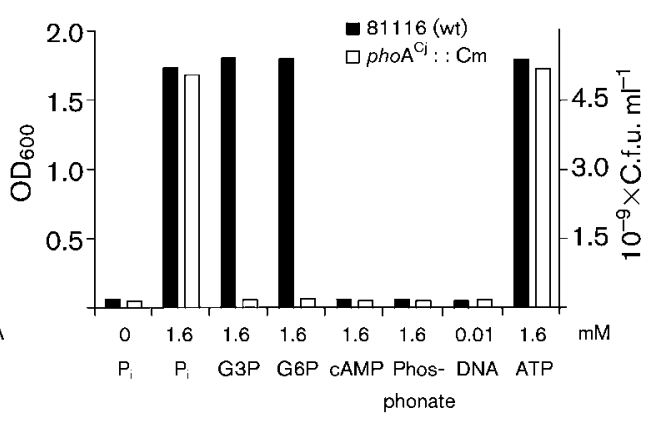

Fig. 2. Biochemical characteristics of $\mathrm{PhoA}^{\mathrm{Cj}}$. (a) The $\mathrm{pH}$ optimum of $\mathrm{PhoA} \mathrm{Cj}^{\mathrm{Cj}}$ activity was determined after lysis of $\mathrm{C}$. jejuni 81116 in buffer of the indicated pH using PNPP as substrate. Standard errors based on four independent experiments are shown. (b) EDTA inhibits $P h o A^{C j}$ activity. PhoA ${ }^{\mathrm{Cj}}$ activity was measured for three C. jejuni strains grown in defined medium containing $0.08 \mathrm{mM} \mathrm{P}_{\mathrm{i}}$. Bacteria were lysed in the presence or absence of $1 \mathrm{mM} \mathrm{EDTA}$ and PhoA ${ }^{\mathrm{Cj}}$ activity was measured using PNPP as substrate. Standard errors based on four independent experiments are shown. (c) $\mathrm{PhoA}^{\mathrm{Cj}}$ requires $\mathrm{Ca}^{2+}$ as cofactor. C. jejuni strain 480 transformed with a plasmid containing $p h o A^{\mathrm{Cj}}$ downstream of the constitutive metK promoter was grown overnight in $\mathrm{HI}$ medium in the absence or presence of EDTA. Then, bacteria were incubated with the indicated cation(s) (each $10 \mathrm{mM}$ ) for $30 \mathrm{~min}$ to monitor their effect on alkaline phosphatase activity. Standard errors based on four independent experiments are shown. (d) Substrate specificity of $\mathrm{PhoA} \mathrm{A}^{\mathrm{Cj}}$. C. jejuni strain 81116 and the pho $A^{\mathrm{Cj}}$ : : Cm mutant were grown overnight in defined medium containing the indicated phosphate sources. Optical densities as well as the corresponding c.f.u. $\mathrm{ml}^{-1}$ are shown. Bacterial cultures were diluted to $\mathrm{OD}_{600}$ between 0.1 and 0.5 to enable accurate measurement of $O D_{600}$. Data correspond to one representative of three independent experiments.

strain carrying the plasmid with the original phoA ${ }^{\mathrm{Cj}}$ (pMA1-PhoA ${ }^{\mathrm{Cj}}$ ), high levels of enzyme activity were present independent of the phosphate concentration in the medium (Fig. 3a). These results strongly suggest that $\mathrm{PhoA}^{\mathrm{Cj}}$ is secreted via a thus far unidentified C. jejuni Tat secretion machinery.

\section{PhoA $^{\mathrm{Cj}_{\mathrm{j}}}$ is transported via the $C$. jejuni Tat secretion system}

Complementary evidence that $\mathrm{PhoA}^{\mathrm{Cj}}$ is secreted via the Tat pathway was sought by inactivation of the system. Genomic analysis suggests that $C$. jejuni has a Tat system, as four of its putative components, tat $A / E$ (Cj1176), tatB (Cj0578), tatC (Cj0579) and tatD (Cj0644), are present in the genome of the sequenced strain NCTC 11168 (Gundogdu et al., 2007; Parkhill et al., 2000) (Fig. 3b).
To test the functionality of the Tat system in C. jejuni, we inactivated the putative tat $C$ homologue by insertion of a kanamycin-resistance cassette. A severe growth defect as measured by optical density and c.f.u. counting was observed for the tatC::Km mutant compared to the wild-type 81116 strain (Fig. 3c). This defect was rectified by introduction of plasmid pMA2-tatC harbouring a constitutively expressed $C$. jejuni tat $C$ gene.

Further evidence of the functionality of the C. jejuni Tat system was obtained by measurement of nitrate reductase activity. A search of the C. jejuni proteome for putative Tat substrates based on the presence of the specific Tat recognition consensus sequence (RRxFLK) (Lee et al., 2006) indicated 11 potential Tat substrates (Table 3) (Dilks et al., 2003), including NapA. In other bacterial species this Tat substrate reduces nitrate to nitrite, which may be used by the bacterium as an alternative electron acceptor 
(a)

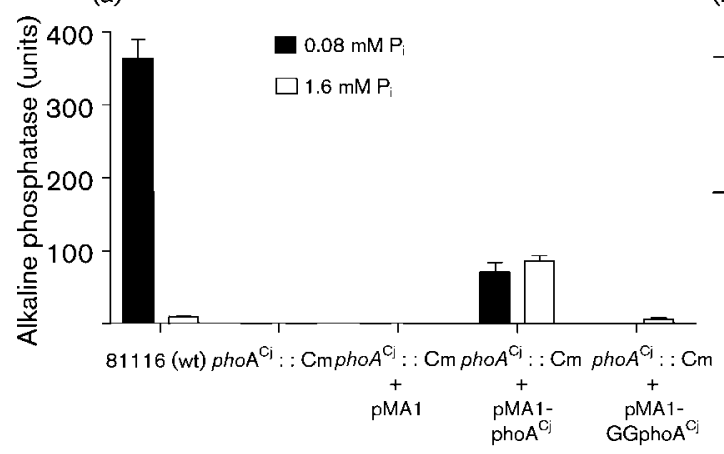

(c)

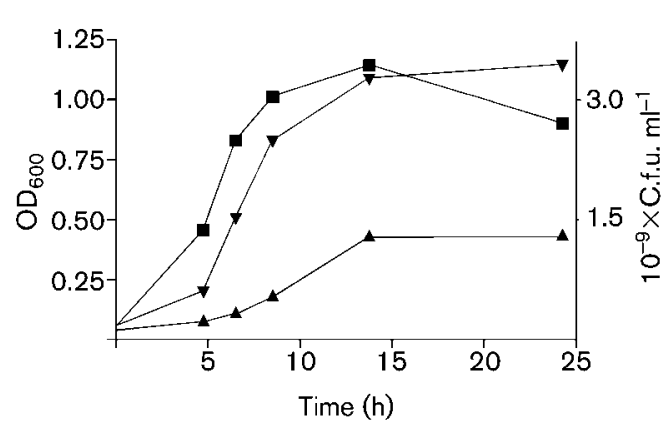

(b)
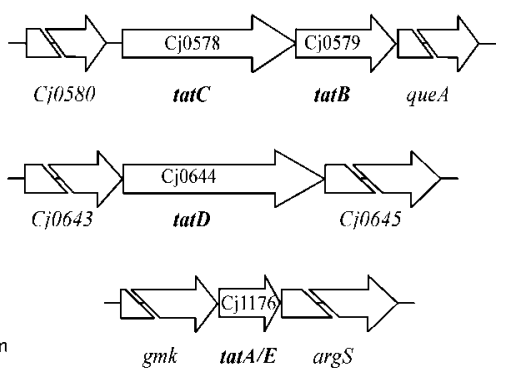

(d)

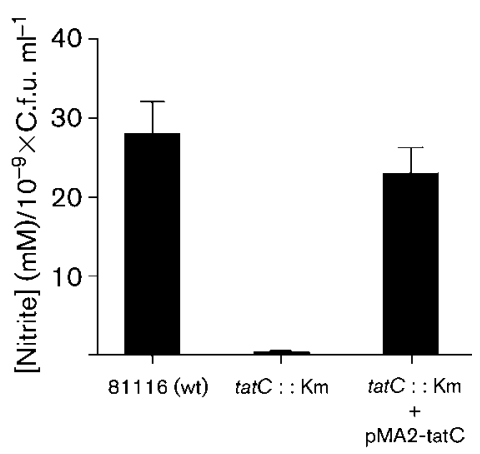

Fig. 3. C. jejuni possesses a functional Tat secretion system. (a) Alkaline phosphatase assay showing that the twin-arginine residues are essential for $\mathrm{Pho} \mathrm{A}^{\mathrm{Cj}}$ activity. Alkaline phosphatase activity was measured for the pho $A^{\mathrm{Cj}}:: \mathrm{Cm}$ mutant, and mutants containing plasmid pMA1 (empty vector), pMA1-phoA ${ }^{\mathrm{Cj}}$ (containing intact PhoA ${ }^{\mathrm{Cj}}$ ) or pMA1-GGpho ${ }^{\mathrm{Cj}}$ (intact $\mathrm{Pho} \mathrm{A}^{\mathrm{Cj}}$ where the twin-arginine residues were replaced by glycine residues) after growth under high- or low-phosphate conditions. (b) Organization of the Tat genes in the genome of $C$. jejuni NCTC 11168. The length of the gene arrows corresponds to the length of the genes. (c) Growth of strain $81116(\boldsymbol{\square})$, the tatC mutant $(\operatorname{tat} C: \mathrm{Km})(\boldsymbol{\Delta})$ and complemented tat $C$ mutant carrying plasmid pMA2-tatC $(\boldsymbol{\nabla})$ in $\mathrm{HI}$ medium under microaerophilic conditions. Growth curves are from one representative of three independent experiments. The optical densities as well as the corresponding c.f.u. $\mathrm{ml}^{-1}$ are presented. Bacterial cultures were diluted to $\mathrm{OD}_{600}$ between 0.1 and 0.5 to enable accurate measurement of $\mathrm{OD}_{600}$. The actual c.f.u. counts fully reflected optical densities for each strain for the entire duration of the incubation, indicating that bacterial growth was measured. (d) Nitrite reductase assay demonstrating that the enzyme required a functional TatC. Nitrite accumulation was measured in culture supernatants of $1 \times 10^{9}$ c.f.u. of C. jejuni strain 81116 , the tatC:: Km mutant and the mutant containing complementation plasmid pMA2-tatC grown in $\mathrm{HI}$ with $50 \mathrm{mM}$ potassium nitrate. Standard errors based on four independent experiments are indicated.

(Pittman \& Kelly, 2005). Analysis of nitrate reductase activity indicated that $C$. jejuni 81116 exhibited this enzyme activity, while the tatC: :Km mutant did not (Fig. 3d). This defect was restored by complementation of the tatC::Km mutant with pMA2-tatC.

To unequivocally demonstrate that $\mathrm{PhoA}^{\mathrm{Cj}}$ is transported via the Tat system, we assessed the phosphatase activity under low-phosphate conditions for the parental strain, the tat $C:: \mathrm{Km}$ mutant and the pMA2-tatC complemented tat $C$ mutant. Inactivation of TatC resulted in very low alkaline phosphatase activity compared to the parent strain. The defect was fully rectified after introduction of an intact copy of the tatC gene (Fig. 4a). To exclude that the lack of enzyme activity in the TatC mutant was caused by a more general protein secretion defect, we introduced into the tat $C:: \mathrm{Km}$ mutant strain a plasmid carrying the $E$. coli PhoA, which utilizes the Sec secretion pathway (Palmer \& Berks, 2003). This enzyme was active in this background, indicating that the Sec system in the $C$. jejuni tat $C$ mutant was still functional (data not shown). These results, in conjunction with the requirement of the twin-arginine motif, indicate that $\mathrm{PhoA}^{\mathrm{Cj}}$ is transported across the cytoplasmic membrane by the Tat secretion pathway. Like the E. coli PhoA, the C. jejuni $\mathrm{PhoA}^{\mathrm{Cj}}$ remains cell associated, as measurement of enzyme activity in the culture supernatant indicated that virtually all the activity remained associated with the cell fractions (Fig. 4b). The 
Table 3. Putative Tat substrates of C. jejuni NCTC 11168

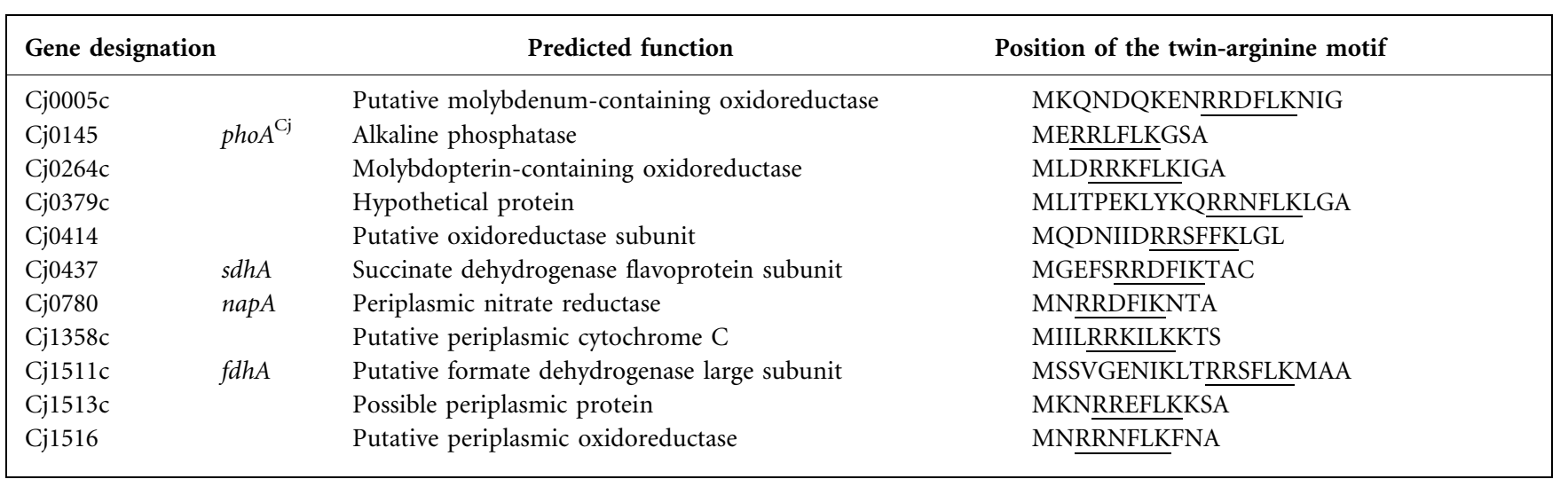

enzyme activity for the E. coli PhoA in $C$. jejuni was primarily found in the periplasmic fraction, while active $C$. jejuni PhoA was mostly found in the membrane fraction.

\section{DISCUSSION}

Most bacterial species contain a number of proteins to assimilate the essential element phosphate $\left(\mathrm{P}_{\mathrm{i}}\right)$ from the environment (Wanner, 1996). Here we provide evidence that the C. jejuni alkaline phosphatase $\left(\mathrm{PhoA}^{\mathrm{Cj}}\right)$ is a key enzyme in the acquisition of $\mathrm{P}_{\mathrm{i}}$. The enzyme differed from the typical PhoA present in most bacterial species as it required calcium as a cofactor and is transported into the periplasm via the Tat secretion pathway. The transport of $\mathrm{PhoA}^{\mathrm{Cj}}$ via the Tat system is the first functional evidence of the existence of this pathway in C. jejuni.

Key evidence that $C$. jejuni Cj0145 encodes the only functional alkaline phosphatase in $C$. jejuni was the successful complementation in trans of the mutant strain with an intact copy of this gene. We placed the intact pho $A^{\mathrm{Cj}}$ gene on a shuttle plasmid under a constitutive promoter rather than its own PhosS-PhosR-regulated promoter to investigate whether additional phosphateregulated factors were required for the enzyme activity. The strong enzyme activity in the complemented strain measured under high-phosphate conditions (Fig. 1) indicates that the $C$. jejuni phosphatase can act independently of other phosphate-regulated molecules. The enzyme utilizes calcium as a cofactor. This became evident when multiple $C$. jejuni strains were tested for phosphatase activity. In some strains, enzyme activity was only observed when EDTA, which was used to lyse the bacteria, was omitted from the lysis buffer or when an excess of calcium was added to the EDTA-containing buffer. The need for a divalent cation as a cofactor is not unusual, as alkaline phosphatases from other bacterial species also require divalent cation(s) to hydrolyse different types of phosphate compounds (Monds et al., 2006; Wang et al., 2005; Wu et al., 2007). E. coli PhoA requires $\mathrm{Mg}^{2+}$ and $\mathrm{Zn}^{2+}$ as cofactors. The requirement of $\mathrm{Ca}^{2+}$ as cofactor has thus far only been reported for $\mathrm{PhoA}^{\mathrm{Vc}}$ of Vibrio cholerae (Roy et al.,
1982) and PhoX of Pasteurella multocida (Wu et al., 2007). In P. multocida an aspartic acid together with a stretch of hydrophobic amino acids near the $\mathrm{C}$ terminus of the protein are essential for $\mathrm{Ca}^{2+}$ binding and phosphatase activity ( $\mathrm{Wu}$ et al., 2007). Analysis of the $\mathrm{PhoA}^{\mathrm{Cj}}$ protein sequence indicates the presence of a similar motif.

Bacterial alkaline phosphatase provides bacteria with $\mathrm{P}_{\mathrm{i}}$ by reducing different phosphoester compounds available in the environment. The alkaline phosphatase of $P$. multocida (Wu et al., 2007) can cleave both phosphomonoester and phosphodiester bonds. Our results indicate that the $C$. jejuni enzyme is able to utilize phosphomonoesters such as glucose 6-phosphate and glycerol 3-phosphate, but not phosphodiesters (Fig. 2d). This may limit the ecological niches of the bacterium.

Alkaline phosphatases generally exert the hydrolysis of phosphomonoester-containing substrates in the bacterial periplasm. E. coli PhoA and most of its homologues in other bacterial species are transported across the cytoplasmic membrane by the main protein secretion system, the Sec system (Palmer \& Berks, 2003). Analysis of the protein sequence of $\mathrm{PhoA}^{\mathrm{Cj}}$ indicated the presence of a typical twin-arginine (Tat) consensus motif in the $\mathrm{N}$ terminus. This RRxFLK motif, in which the twin-arginine is highly conserved (Stanley et al., 2000), led to the discovery that the $C$. jejuni phosphatase exploits the Tat secretion machinery to gain access to the periplasm. Evidence that the Tat system serves as transport machinery for $\mathrm{PhoA}^{\mathrm{Cj}}$ includes the lack of enzyme activity after substitution of the twin-arginine residues (Fig. 3a) and after inactivation of the tatC gene, which results in dysfunction of the Tat system (Fig. 4a). The absence of enzyme activity in these strains even in the presence of calcium (data not shown) implies that the enzyme is not active in the cytosol, consistent with its assumed function. Although $p h o A^{\mathrm{Cj}}$ was transcribed, $\mathrm{PhoA}^{\mathrm{Cj}}$ is probably not transported through the E. coli Tat system, because no difference was observed in alkaline phosphatase activity between $E$. coli wild-type and a tat $C$ mutant (data not shown). Furthermore, the $E$. coli tat $C$ mutant could not be complemented with an intact C. jejuni tatC (data not shown). This may indicate that, in 
(a)

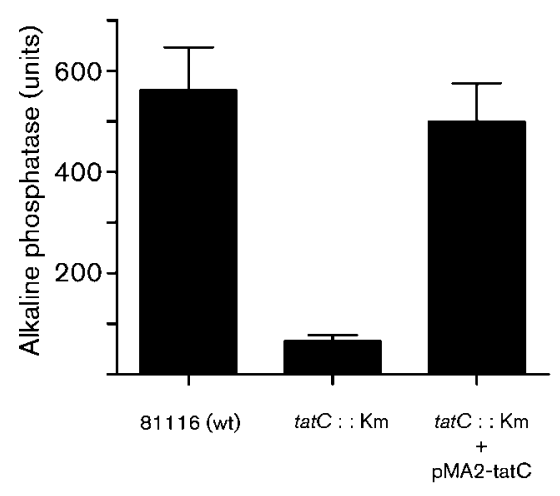

(b)

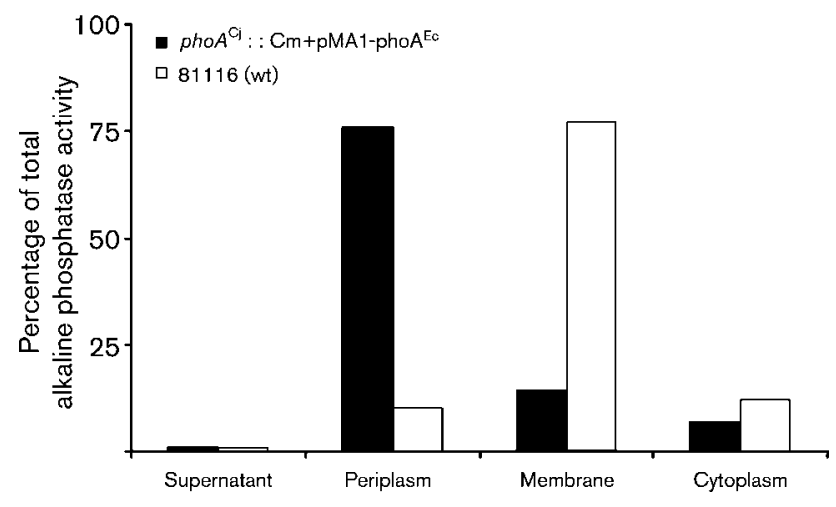

Fig. 4. Localization of $P h o A^{C j}$ and the role of the C. jejuni Tat secretion system. (a) Alkaline phosphatase assay demonstrating that $\mathrm{PhoA} \mathrm{A}^{\mathrm{Cj}}$ requires a functional TatC. $\mathrm{PhoA}^{\mathrm{Cj}}$ activities for $\mathrm{C}$. jejuni strain 81116, the tatC::Km mutant and the mutant complemented with plasmid pMA2-tatC were determined after $8 \mathrm{~h}$ growth in defined medium containing $0.08 \mathrm{mM} \mathrm{P}_{\mathrm{i}}$. Standard errors based on four replicates are shown. (b) Alkaline phosphatase assay showing that $P h o A^{C j}$ is associated with the cellular fraction. PhoA ${ }^{\mathrm{Cj}}$ activities for strain 81116 and the $p h o A^{\mathrm{Cj}}:: \mathrm{Cm}$ mutant complemented with plasmid pMA1-phoA ${ }^{\mathrm{Ec}}$ containing the $E$. coli pho $A$ were measured after overnight growth in defined medium containing $0.08 \mathrm{mM} \mathrm{P}_{\mathrm{i}}$. The culture supernatant, periplasm, cytoplasm and membrane were separated to determine the localization of active alkaline phosphatase. The values corresponding to $100 \%$ were 386 units of alkaline phosphatase for 81116 and 120 units for the phoA::Cm mutant containing pMA1-phoA ${ }^{\mathrm{Ec}}$. The experiment was repeated four times with similar results.

contrast to the Sec systems, at least some components of the Tat systems of $C$. jejuni and E. coli are not compatible.

The transport of $\mathrm{PhoA}^{\mathrm{Cj}}$ via the Tat system is the first reported evidence that this secretion pathway is functional in $C$. jejuni. Genome analysis predicts at least 11 putative Tat substrates in C. jejuni strain NCTC 11168 (Table 3) (Dilks et al., 2003). Besides $\mathrm{PhoA}^{\mathrm{Cj}}$, we provide evidence that nitrate reductase NapA, a well-documented Tat substrate in other bacterial species (Ding \& Christie, 2003; Lavander et al., 2006; Weiner et al., 1998), requires the Tat system of C. jejuni (Fig. 3d). The lack of nitrate reductase activity in the TatC mutant but gain-of-function after introduction of an intact copy of the gene clearly demonstrates that $\mathrm{Tat} C$, which is an essential component of the Tat system (Bogsch et al., 1998; Jongbloed et al., 2000), is required for NapA function. Considering the nature of most predicted Tat substrates, which are mostly cofactor requiring enzymes involved in electron transport (Table 3), it seems plausible to assume that the main function of the Tat secretion system in C. jejuni is to translocate redox cofactor-containing proteins that contribute to the assembly of the electron transport chain and energy conservation under oxygen-limited conditions (Palmer \& Berks, 2003). Why the alkaline phosphatase of C. jejuni also exploits this secretion pathway, rather than the classical Sec system often used by PhoA of other species, awaits further investigation.

The atypical use of the Tat system to transport alkaline phosphatase across the cytoplasmic membrane has also been demonstrated for Pseudomonas fluorescens and Thermus thermophilus (Monds et al., 2006; Angelini et al., 2001) and is assumed for V. cholerae and P. multocida (Wu et al., 2007). P. fluorescens, however, also possesses Tatindependent alkaline phosphatases (Monds et al., 2006). Interestingly, the sole alkaline phosphatase $\mathrm{PhoA}^{\mathrm{Cj}}$ of $C$. jejuni is $52 \%$ identical to the $V$. cholerae $\mathrm{PhoA}^{\mathrm{Vc}}$ protein, and $49 \%$ and $41 \%$ identical to the PhoX proteins of $P$. fluorescens and $P$. multocida, respectively. Furthermore, $\mathrm{PhoA}^{\mathrm{Vc}}$ of $V$. cholerae and PhoX of $P$. multocida are activated by $\mathrm{Ca}^{2+}$, as we found for the C. jejuni enzyme. Similarly, the pH optimum of $\mathrm{PhoA}^{\mathrm{Cj}}(\sim \mathrm{pH} 10$, Fig. 2a) is comparable to that of the $P$. multocida homologue $(\mathrm{Wu}$ et al., 2007), which is higher than that for E. coli PhoA ( $\mathrm{pH}$ 8.5) (Nesmeyanova et al., 1981). Because of their shared dependence on the Tat secretion system, the alkaline phosphatases of $V$. cholerae, $P$. fluorescens and $P$. multocida have been proposed to form a separate group of enzymes, designated the PhoX family ( $\mathrm{Wu}$ et al., 2007). The sequence similarity and functional characteristics of the C. jejuni $\mathrm{PhoA}^{\mathrm{Cj}}$ indicate that $\mathrm{PhoA}^{\mathrm{Cj}}$ also belongs to this group and therefore we propose to rename $\mathrm{PhoA}^{\mathrm{Cj}}$ as PhoX. This further supports the designation of a novel PhoX family of Tat-dependent alkaline phosphatases.

\section{ACKNOWLEDGEMENTS}

We thank Professor Dr T. Palmer for providing us the E. coli strains

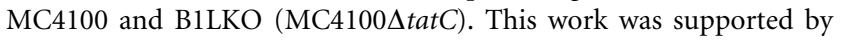
NWO-VIDI grant 917.66 .330 to M. M. S. M.W.

\section{REFERENCES}

Altekruse, S. F., Stern, N. J., Fields, P. I. \& Swerdlow, D. L. (1999). Campylobacter jejuni - an emerging foodborne pathogen. Emerg Infect Dis 5, 28-35.

Angelini, S., Moreno, R., Gouffi, K., Santini, C., Yamagishi, A., Berenguer, J. \& Wu, L. (2001). Export of Thermus thermophilus 
alkaline phosphatase via the twin-arginine translocation pathway in Escherichia coli. FEBS Lett 506, 103-107.

Bogsch, E. G., Sargent, F., Stanley, N. R., Berks, B. C., Robinson, C. \& Palmer, T. (1998). An essential component of a novel bacterial protein export system with homologues in plastids and mitochondria. J Biol Chem 273, 18003-18006.

Diergaardt, S. M., Venter, S. N., Spreeth, A., Theron, J. \& Brozel, V. S. (2004). The occurrence of campylobacters in water sources in South Africa. Water Res 38, 2589-2595.

Dilks, K., Rose, R. W., Hartmann, E. \& Pohlschroder, M. (2003). Prokaryotic utilization of the twin-arginine translocation pathway: a genomic survey. J Bacteriol 185, 1478-1483.

Ding, Z. \& Christie, P. J. (2003). Agrobacterium tumefaciens twinarginine-dependent translocation is important for virulence, flagellation, and chemotaxis but not type IV secretion. J Bacteriol 185, 760-771.

Gundogdu, O., Bentley, S. D., Holden, M. T., Parkhill, J., Dorrell, N. \& Wren, B. W. (2007). Re-annotation and re-analysis of the Campylobacter jejuni NCTC11168 genome sequence. BMC Genomics 8, 162.

Jongbloed, J. D., Martin, U., Antelmann, H., Hecker, M., Tjalsma, H., Venema, G., Bron, S., van Dijl, J. M. \& Muller, J. (2000). TatC is a specificity determinant for protein secretion via the twin-arginine translocation pathway. J Biol Chem 275, 41350-41357.

Labigne-Roussel, A., Harel, J. \& Tompkins, L. (1987). Gene transfer from Escherichia coli to Campylobacter species: development of shuttle vectors for genetic analysis of Campylobacter jejuni.J Bacteriol 169, $5320-5323$.

Lavander, M., Ericsson, S. K., Broms, J. E. \& Forsberg, A. (2006). The twin arginine translocation system is essential for virulence of Yersinia pseudotuberculosis. Infect Immun 74, 1768-1776.

Leach, S., Harvey, P. \& Wali, R. (1997). Changes with growth rate in the membrane lipid composition of and amino acid utilization by continuous cultures of Campylobacter jejuni. J Appl Microbiol 82, 631-640.

Lee, P. A., Tullman-Ercek, D. \& Georgiou, G. (2006). The bacterial twin-arginine translocation pathway. Annu Rev Microbiol 60, 373-395.

MacKichan, J. K., Gaynor, E. C., Chang, C., Cawthraw, S., Newell, D. G., Miller, J. F. \& Falkow, S. (2004). The Campylobacter jejuni dccRS two-component system is required for optimal in vivo colonization but is dispensable for in vitro growth. Mol Microbiol 54, 1269-1286.

Miller, W. G., Bates, A. H., Horn, S. T., Brandl, M. T., Wachtel, M. R. \& Mandrell, R. E. (2000). Detection on surfaces and in Caco-2 cells of Campylobacter jejuni cells transformed with new $g f p, y f p$, and $c f p$ marker plasmids. Appl Environ Microbiol 66, 5426-5436.

Monds, R. D., Newell, P. D., Schwartzman, J. A. \& O'Toole, G. A. (2006). Conservation of the Pho regulon in Pseudomonas fluorescens Pf0-1. Appl Environ Microbiol 72, 1910-1924.

Myers, J. D. \& Kelly, J. K. (2005). A sulphite respiration system in the chemoheterotrophic human pathogen Campylobacter jejuni. Microbiology 151, 233-242.

Nesmeyanova, M. A., Motlokh, O. B., Kolot, M. N. \& Kulaev, I. S. (1981). Multiple forms of alkaline phosphatase from Escherichia coli cells with repressed and derepressed biosynthesis of the enzyme. J Bacteriol 146, 453-459.

Palmer, T. \& Berks, B. C. (2003). Moving folded proteins across the bacterial cell membrane. Microbiology 149, 547-556.
Palmer, S. R., Gully, P. R., White, J. M., Pearson, A. D., Suckling, W. G., Jones, D. M., Rawes, J. C. \& Penner, J. L. (1983). Water-borne outbreak of campylobacter gastroenteritis. Lancet 1, 287-290.

Parkhill, J., Wren, B. W., Mungall, K., Ketley, J. M., Churcher, C., Basham, D., Chillingworth, T., Davies, R. M., Feltwell, T. \& other authors (2000). The genome sequence of the food-borne pathogen Campylobacter jejuni reveals hypervariable sequences. Nature 403, 665-668.

Pittman, M. S. \& Kelly, D. J. (2005). Electron transport through nitrate and nitrite reductases in Campylobacter jejuni. Biochem Soc Trans 33, 190-192.

Rosef, O., Rettedal, G. \& Lageide, L. (2001). Thermophilic campylobacters in surface water: a potential risk of campylobacteriosis. Int J Environ Health Res 11, 321-327.

Roy, N. K., Ghosh, R. K. \& Das, J. (1982). Monomeric alkaline phosphatase of Vibrio cholerae. J Bacteriol 150, 1033-1039.

Schneider, K. \& Beck, C. F. (1986). Promoter-probe vectors for the analysis of divergently arranged promoters. Gene 42 , 37-48.

Sellars, M. J., Hall, S. J. \& Kelly, D. J. (2002). Growth of Campylobacter jejuni supported by respiration of fumarate, nitrate, nitrite, trimethylamine- $N$-oxide, or dimethyl sulfoxide requires oxygen. $J$ Bacteriol 184, 4187-4196.

Stanley, N. R., Palmer, T. \& Berks, B. C. (2000). The twin arginine consensus motif of Tat signal peptides is involved in Sec-independent protein targeting in Escherichia coli. J Biol Chem 275, 11591-11596.

Torriani, A. (1990). From cell membrane to nucleotides: the phosphate regulon in Escherichia coli. Bioessays 12, 371-376.

van Vliet, A. H., Wooldridge, K. G. \& Ketley, J. M. (1998). Ironresponsive gene regulation in a Campylobacter jejuni fur mutant. J Bacteriol 180, 5291-5298.

Wang, J., Stieglitz, K. A. \& Kantrowitz, E. R. (2005). Metal specificity is correlated with two crucial active site residues in Escherichia coli alkaline phosphatase. Biochemistry 44, 8378-8386.

Wanner, B. L. (1996). Phosphorus assimilation and control of the phosphate regulon. In Escherichia coli and Salmonella: Cellular and Molecular Biology, pp. 1357-1381. Edited by F. C. Neidhardt and others. Washington, DC: American Society for Microbiology.

Wassenaar, T. M., Fry, B. N. \& van der Zeijst, B. A. (1993). Genetic manipulation of Campylobacter: evaluation of natural transformation and electro-transformation. Gene 132, 131-135.

Weiner, J. H., Bilous, P. T., Shaw, G. M., Lubitz, S. P., Frost, L., Thomas, G. H., Cole, J. A. \& Turner, R. J. (1998). A novel and ubiquitous system for membrane targeting and secretion of cofactorcontaining proteins. Cell 93, 93-101.

Wösten, M. M., Parker, C. T., van Mourik, A., Guilhabert, M. R., van Dijk, L. \& van Putten, J. P. (2006). The Campylobacter jejuni PhosS/ PhosR operon represents a non-classical phosphate-sensitive twocomponent system. Mol Microbiol 62, 278-291.

Wu, J. R., Shien, J. H., Shieh, H. K., Hu, C. C., Gong, S. R., Chen, L. Y. \& Chang, P. C. (2007). Cloning of the gene and characterization of the enzymatic properties of the monomeric alkaline phosphatase (PhoX) from Pasteurella multocida strain X-73. FEMS Microbiol Lett 267, $113-120$.

Edited by: J. M. Becker 